Review Article

\title{
Clinical Significance of C-Reactive Protein to Albumin Ratio in Patients with Hepatocellular Carcinoma: A Meta-Analysis
}

\author{
Nanping Lin $\mathbb{D}^{1},{ }^{1,2}$ Jingrong $\mathrm{Li}^{3}{ }^{3}$ Qiao Ke, ${ }^{1,2}$ Lei Wang $\mathbb{D},{ }^{1,4}$ Yingping Cao, ${ }^{3}$ \\ and Jingfeng Liu $\mathbb{1 D}^{1,2}$ \\ ${ }^{1}$ Department of Hepatopancreatobiliary Surgery, Mengchao Hepatobiliary Hospital of Fujian Medical University, Fuzhou, \\ Fujian, China \\ ${ }^{2}$ The First Affiliated Hospital of Fujian Medical University, Fuzhou, Fujian, China \\ ${ }^{3}$ Department of laboratory, Fujian Medical University Union Hospital, Fuzhou, Fujian, China \\ ${ }^{4}$ Department of Radiation Oncology, Mengchao Hepatobiliary Hospital of Fujian Medical University, Fuzhou, Fujian, China
}

Correspondence should be addressed to Lei Wang; wangleiy001@sina.com and Jingfeng Liu; drjingfeng@126.com

Received 18 September 2019; Accepted 4 August 2020; Published 3 September 2020

Academic Editor: Carlo Chiarla

Copyright (C) 2020 Nanping Lin et al. This is an open access article distributed under the Creative Commons Attribution License, which permits unrestricted use, distribution, and reproduction in any medium, provided the original work is properly cited.

\begin{abstract}
Aim. To evaluate the prognostic significance of C-reactive protein to albumin ratio (CAR) for clinical outcomes in hepatocellular carcinoma (HCC) patients. Material and Methods. Eligible studies were searched by PubMed, MedLine, the Cochrane Library, from January 1, 2000, to June 30,2019, investigating the prognostic value of CAR in patients with HCC. Primary endpoint was OS. Hazard ratio (HR) with 95\% confidence interval (CI) was used to determine the effect size. Results. 7 records including 2208 patients published since 2014 were enrolled into our meta-analysis. Clinicopathological characteristics were also correlated with the level of CAR. The pooled HR for the OS rate between low and high CAR groups was 2.13 (95\% CI $1.70 \sim 2.68, P<0.00001$ ) using a random model, but sensitivity analysis showed that the pooled HR for the OS rates did not change substantially after removal of any included study. As for patients receiving surgery, the pooled HR for the OS rate between low and high CAR groups was 2.04 (95\% CI 1.59 2.61, $P<0.00001)$. Subgroup analysis showed that CAR could be a prognostic biomarker for HCC patients regardless of regions (China, $\mathrm{HR}=1.75,95 \%$ CI 1.51 2.02; Japan, HR =3.36, 95\% CI 2.07 5.45; Korea, HR= 2.26, 95\% CI 1.47 4.47; respectively), the cut-off value $(<0.1, \mathrm{HR}=2.84,95 \%$ CI 1.90 4.24; >0.1, HR = 1.99, 95\% CI 1.52 2.61; respectively), and sample size $(<200, \mathrm{HR}=2.85,95 \% \mathrm{CI} 2.01 \sim 4.03 ;>200, \mathrm{HR}=1.75,95 \%$ CI $1.52 \sim 2.02$; respectively). Conclusion. With the current data, we clearly concluded that CAR was closely correlated with prognosis of patients with HCC. Multicenter, prospective randomized trials are warranted to confirm the conclusion.
\end{abstract}

\section{Introduction}

The incidence of hepatocellular carcinoma (HCC) is increasing stably worldwide, but the prognosis still far from satisfactory [1]. Radical resection is still one of the most efficient strategies to cure HCC, but the incidence of recurrence at 5 -year is reported to be as high as $70-80 \%[2,3]$. Considering $80 \%$ of patients have lost the chance of surgery at diagnosis [4], strategies for HCC varied from different stages $[5,6]$. Hence, biomarkers served as predictors of prognosis and aid of decision-making are badly needed in clinical.

C-reactive protein to albumin ratio (CAR) has been reported as a powerful prognostic indicator for solid tumors
[7-9], and it has been confirmed in colorectal cancer, esophageal cancer, and nasopharyngeal cancer by several newly published meta-analysis [10-12]. Reasons might be as follows: (1) many of the solid tumors are highly associated with inflammation, and C-reactive protein (CRP) is one of the most common kinds of systematic inflammatory index; (2) nutritional status is one of the crucial factors for the longterm prognosis of patients with cancers, which is attracting more and more attentions, and albumin (ALB) level is the simplest marker to evaluate the status of nutrition.

Recently, high CAR has been reported to be correlated with poor prognosis of patients with HCC [13-19], but the results varied from each other. And to the best of our 
knowledge, there are no meta-analysis and systematic review evaluating the prognostic value of CAR in patients with HCC. Therefore, a meta-analysis was warranted to determine the prognostic significance of CAR for clinical outcomes in HCC patients.

\section{Material and Method}

This study was designed according to PICOS principles and conducted based on the preferred Reporting Items for Systematic Reviews and Meta-Analyses (PRISMA) statement [20].

2.1. Literature Search. A comprehensive search on the existing published medical literature was conducted by Jingrong $\mathrm{Li}$ and Nanping Lin to investigate the prognostic value of CAR for patients with HCC. English electronic databases such as PubMed, MedLine, and Embase were used to search the literature from January 1, 2000, to June 30, 2019. Key words were as follows: (("hepatocellular" or "liver" or "hepatic") AND "tumour" or "tummor" or "cancer" or "carcinoma" or "neoplasm") OR ("HCC” or "LC")) AND (“C-reactive protein" or "albumin" or "CAR"). Any potentially eligible studies were then identified manually through the references of the included studies, reviews, letters, and comments [21].

2.2. Selection Criteria. Inclusion criteria: (i) patients with clinic or pathological confirmed HCC; (ii) pretreatment CRP and albumin was determined; (iii) clinical outcomes including overall survival (OS), disease-free survival (DFS).

Exclusion criteria: (i) patients including benign disease or other tumors; (ii) only CRP or albumin was detected before any treatment; (iii) patients showed clinical evidence of inflammatory conditions rather than hepatitis were also excluded; (iv) data on the clinical outcomes was not available; (v) in vivo studies; (vi) conference abstracts, reviews, letters, and comments.

2.3. Endpoints. Primary endpoint was OS. Secondary endpoints were DFS.

2.4. Data Extraction. Data such as the author's information, year of publication, patient's basic characteristic, cut-off value, follow-up time, and outcomes were extracted and assessed by Jingrong Li and Nanping Lin with predefined forms. The hazard ratios (HRs) of OS were extracted directedly from the original data or extracted from the Kaplan-Meier curves according to the methods described in detail by Tierney et al. and Parmar et al. In case of disagreement, a third investigator, Qiao Ke, was intervened to reach a conclusion [21].

2.5. Quality Assessment. The quality of nonrandomized studies was assessed by the modified Newcastle-Ottawa Scale (NOS), and more than 6 stars were defined as high quality, 4 6 stars as medium quality, and less than 4 stars as low quality.

2.6. Statistical Analysis. The meta-analysis was registered at http://www.crd.york.ac.uk/PROSPERO/ (Review registry 143152) and was performed using RevMan Version 5.3. The pooled HRs for OS between high and low levels of CAR were evaluated with $95 \%$ Cis. The effects mode that was used depended on the heterogeneity, which was assessed by the $\chi^{2}$ test and $I^{2}$ statistics; $P<0.10$ or $I^{2}>50 \%$ were considered as significant heterogeneity, and random-effected was chosen. When the hypothesis of homogeneity was rejected, the fixed-effects model was used to estimate the case with homogeneity. Sensitivity analysis was conducted as follows: one study at a time was removed, and the remained were reanalyzed to determine whether the results could be affected significantly by single study. Begg's and Egger's tests were used to evaluate publication bias using Stata 14. Trim and filling method was used to evaluate the stability of the result if $P<0.05$ [21].

\section{Results}

3.1. Base Characteristic of the Included Studies. Totally, 202 records were identified by Jingrong Li and Nanping Lin. 9 records were excluded for duplication by NoteExpress 3.1, and then, 186 records were excluded after browsing titles and abstracts. Hence, 7 records [13-19] including 202 patients published since 2014 were enrolled into our meta-analysis. The search results and details were shown in Figure 1.

The characteristics and baseline demographic data of the patients in each research were listed in Table 1. Of note, ALB to CRP ratio was reported in one study [19] and transferred it into CAR accordingly, which was confirmed repeatedly by Jingrong Li, Nanping Lin, and Qiao Ke. All studies were scored above 6 by NOS.

Clinicopathological characteristics were also correlated with the level of CAR. As shown in Table 2, the mean level of AFP in the high group was higher than that in the low group $(28-38.5 \mathrm{ng} / \mathrm{ml}$ vs $8-28 \mathrm{ng} / \mathrm{ml})$, and the tumor in the high group was bigger than that in the low group (1.0$20.0 \mathrm{~cm}$ vs $0.7-5.2 \mathrm{~cm}$ ). As shown in Table 3, high CAR was found to be with multiply tumors and advanced TNM stage.

3.2. Primary Endpoint. The OS rates comparing between low and high CAR groups were evaluated in 7 included studies [13-19]. Heterogeneities were observed $\left(I^{2}=45 \%, P=0.09\right)$, and using a random model the pooled HR for the OS rate between low and high CAR groups was 2.13 (95\% CI 1.70 2.68, $P<0.00001$, Figure 2(a)).

Heterogeneities disappeared after removing Chen's study [17] $\left(I^{2}=0, P=0.44\right)$, and then, the pooled HR for the OS rate between low and high CAR groups was 2.31 (95\% CI 1.87 2.84, $P<0.00001$, Figure 2(b)) using a fixed model, which indicated that the results were considerably reliable.

3.3. Subgroup Analysis of Hepatectomy for HCC. The OS rates of HCC patients receiving hepatectomy comparing between low and high CAR groups were evaluated in four included studies $[15,16,18,19]$. Significant heterogeneity was not observed $\left(I^{2}=0, P=0.76\right)$, and using a fixed model. The pooled HR for the OS rate between low and high CAR groups was 2.04 (95\% CI 1.59 2.61, $P<0.00001$, Figure 3(a)).

The DFS rates of HCC patients receiving hepatectomy comparing between low and high CAR groups were evaluated in three included studies $[15,16,19]$. Significant 


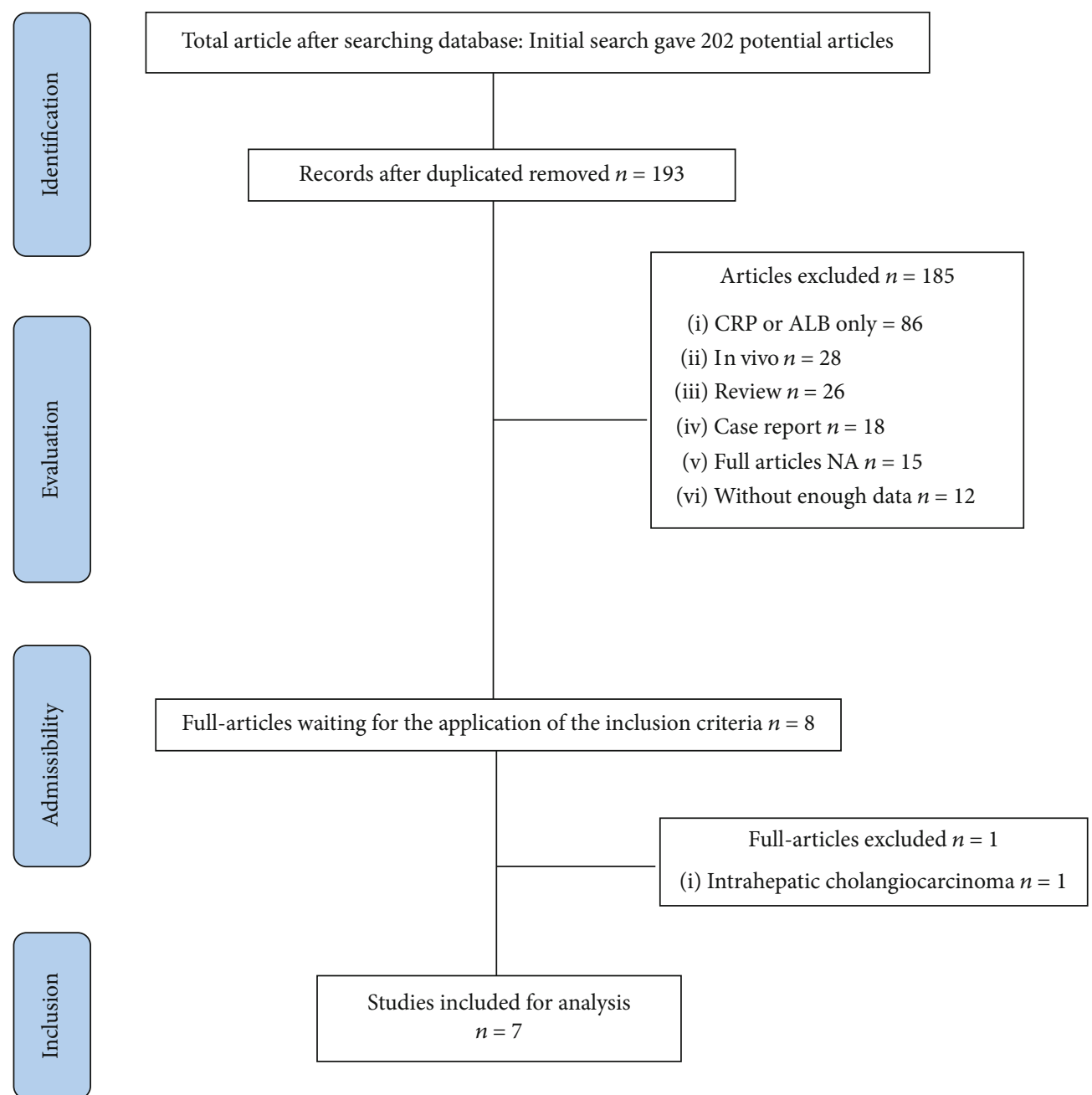

FIGURE 1: PRISMA flow diagram showing selection of articles for meta-analysis.

TABLE 1: Basic characteristic of the included article.

\begin{tabular}{|c|c|c|c|c|c|c|c|c|c|}
\hline Studies & Country & Study year & $\begin{array}{c}\text { Tumor } \\
\text { type }\end{array}$ & $\begin{array}{l}\text { Cut-off } \\
\text { value }\end{array}$ & $\begin{array}{c}\text { Study sample } \\
(\mathrm{L} / \mathrm{H})\end{array}$ & $\begin{array}{l}\text { Received } \\
\text { therapy }\end{array}$ & $\begin{array}{l}\text { Follow-up time } \\
\text { (month) }\end{array}$ & $\begin{array}{c}\text { Prime } \\
\text { endpoint }\end{array}$ & $\begin{array}{l}\text { NOS } \\
\text { score }\end{array}$ \\
\hline Kinoshita 2014 & Japan & $2005-2012$ & HCC & 0.037 & $186(84 / 102)$ & Multiply & $18(1-88)$ & OS & 7 \\
\hline Pang 2017 & China & $2007-2014$ & $\mathrm{HCC}$ & - & 139 & Surgery & $23.1(0.4-103.2)$ & OS/RFS & 7 \\
\hline Shimizu 2017 & Japan & $2006-2013$ & $\mathrm{HCC}$ & 0.028 & $239(84 / 155)$ & Surgery & - & OS/RFS & 8 \\
\hline Oh 2018 & Korea & $2004-2013$ & $\mathrm{HCC}$ & 0.625 & 389 & Surgery & - & OS/RFS & 7 \\
\hline Ren 2018 & China & $2012-2017$ & $\mathrm{HCC}$ & 0.037 & $187(95 / 92)$ & Multiply & $23(1-60)$ & OS/TFS & 8 \\
\hline Chen 2018 & China & $2013-2016$ & $\mathrm{HCC}$ & - & 659 & - & $21.6(1-52.7)$ & OS & 6 \\
\hline Wu 2019 & China & $2008-2012$ & $\mathrm{HCC}$ & 0.185 & $409(236 / 173)$ & Multiply & Until Jun 302016 & OS & 8 \\
\hline
\end{tabular}

HCC: hepatocellular carcinoma; NOS: Newcastle-Ottawa Scale; “-”: not mentioned.

heterogeneity was not observed $\left(I^{2}=0, P=0.73\right)$; then, fixed model was selected. The pooled HR for the OS rate between low and high CAR groups was 1.65 (95\% CI 1.35 2.02, $P<$ 0.00001 , Figure 3(b)).

\subsection{Subgroup Analysis of the Correlation between CAR and} OS. As summarized in Table 4, subgroup analysis of OS stratified by regions, the cut-off value for CAR, and the sample size were conducted. Results showed that CAR could be a prognostic biomarker for HCC patients regardless of regions (China, HR = 1.75, 95\% CI 1.51 2.02; Japan, HR = 3.36, 95\% CI 2.07 5.45; Korea, HR = 2.26, 95\% CI 1.47 4.47; respectively), the cut-off value $(<0.1, \mathrm{HR}=2.84,95 \% \mathrm{CI}$ 1.90 4.24; >0.1, HR = 1.99, 95\% CI 1.52 2.61; respectively), and sample size $(<200, \mathrm{HR}=2.85,95 \%$ CI 2.01 4.03; >200, $\mathrm{HR}=1.75,95 \%$ CI 1.52 2.02; respectively). 
TABLE 2: Correlations between low and high CAR groups with clinicopathological characteristics unable to conduct with meta-analysis.

\begin{tabular}{lcccccc}
\hline \multirow{2}{*}{ Studies factor } & \multicolumn{2}{c}{ Kinosta 2014} & \multicolumn{2}{c}{ Shimizu 2017 } & \multicolumn{2}{c}{ Ren 2018 } \\
& Low & High & Low & High & Low & High \\
\hline AFP $(\mu \mathrm{g} / \mathrm{L})$ (mean and range) & $17.7(2-1,693)$ & $36(1-280,6)$ & $8(4-85)$ & $28(6-337)$ & $28(19-49)$ & $38.5(25.3-56.0)$ \\
Maximum tumor size $(\mathrm{cm})$ & $2.5(0.7-8.8)$ & $4.3(1.0-20.0)$ & $2.5(1.8-3.7)$ & $4.0(2.3-6.5)$ & $3.5(2.7-5.2)$ & $6.0(4.34-9.5)$ \\
\hline
\end{tabular}

TABLE 3: Correlations between low and high CAR groups with clinicopathological characteristics conducted with meta-analysis.

\begin{tabular}{lccccc}
\hline Items factors & Included studies & OR (95\% CI) & $P$ value & $I^{2}$ & Analysis model \\
\hline Liver cirrhosis & 2 & $1.07(0.72,1.59)$ & 0.75 & $0 \%$ & Fixed \\
Multiply tumors & 3 & $0.57(0.69,0.84)$ & 0.005 & $17 \%$ & $85 \%$ \\
Child grade A & 2 & $0.61(0.20,1.91)$ & 0.40 & $63 \%$ & Rixed \\
MVI & 2 & $0.56(0.25,1.27)$ & 0.17 & Random \\
TNM I, II, III, and IV & 3 & $0.43(0.25,0.72)$ & 0.001 & $46 \%$ & Fixed \\
\hline
\end{tabular}



FIGURE 2: Forest plot of the pooled HR for the OS rates comparing between low and high CAR groups.

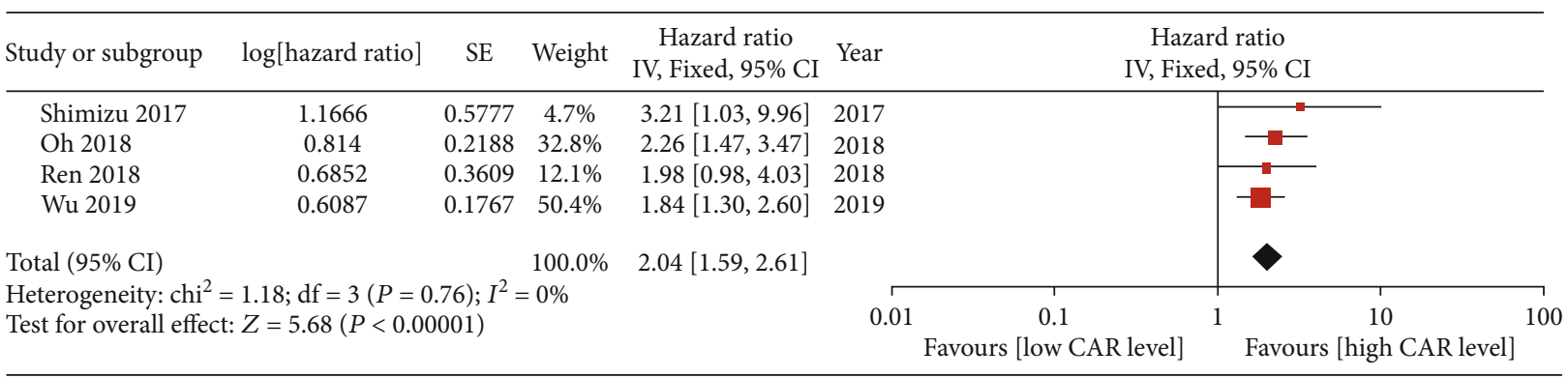

(a)

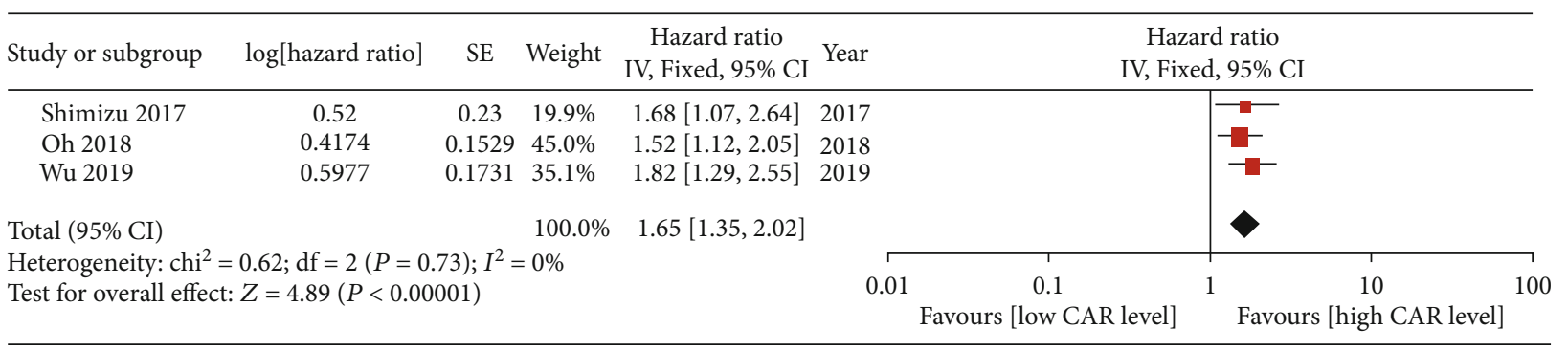

(b)

FIGURE 3: Subgroup analysis of hepatectomy for HCC. (a) Forest plot of the pooled HR for the OS rates comparing between low and high CAR groups. (b) Forest plot of the pooled HR for the DFS rates comparing between low and high CAR groups. 
TABLE 4: Subgroup analysis of the correlation between CAR and OS in different factors.

\begin{tabular}{|c|c|c|c|c|c|c|}
\hline \multirow{2}{*}{ Subgroups } & \multirow{2}{*}{ Included studies } & \multirow{2}{*}{ Pooled HR value } & \multirow{2}{*}{$95 \%$ confidence interval } & \multirow{2}{*}{$P$ value } & \multicolumn{2}{|c|}{ Heterogeneity } \\
\hline & & & & & $I^{2}$ & $P$ value \\
\hline \multicolumn{7}{|l|}{ Region } \\
\hline China & 4 & 1.75 & $1.51,2.02$ & $<0.001$ & 20 & 0.29 \\
\hline Japan & 2 & 3.36 & $2.07,5.45$ & $<0.001$ & 0 & 0.93 \\
\hline Korea & 1 & 2.26 & $1.47,4.47$ & $<0.001$ & - & - \\
\hline \multicolumn{7}{|c|}{ Cut-off value } \\
\hline$<0.1$ & 3 & 2.84 & $1.90,4.24$ & $<0.001$ & 0 & 0.48 \\
\hline$>0.1$ & 2 & 1.99 & $1.52,2.61$ & $<0.001$ & 0 & 0.47 \\
\hline \multicolumn{7}{|l|}{ Sample } \\
\hline$<200$ & 3 & 2.85 & $2.01,4.03$ & $<0.001$ & 0 & 0.49 \\
\hline$>200$ & 4 & 1.75 & $1.52,2.02$ & $<0.001$ & 3 & 0.38 \\
\hline
\end{tabular}

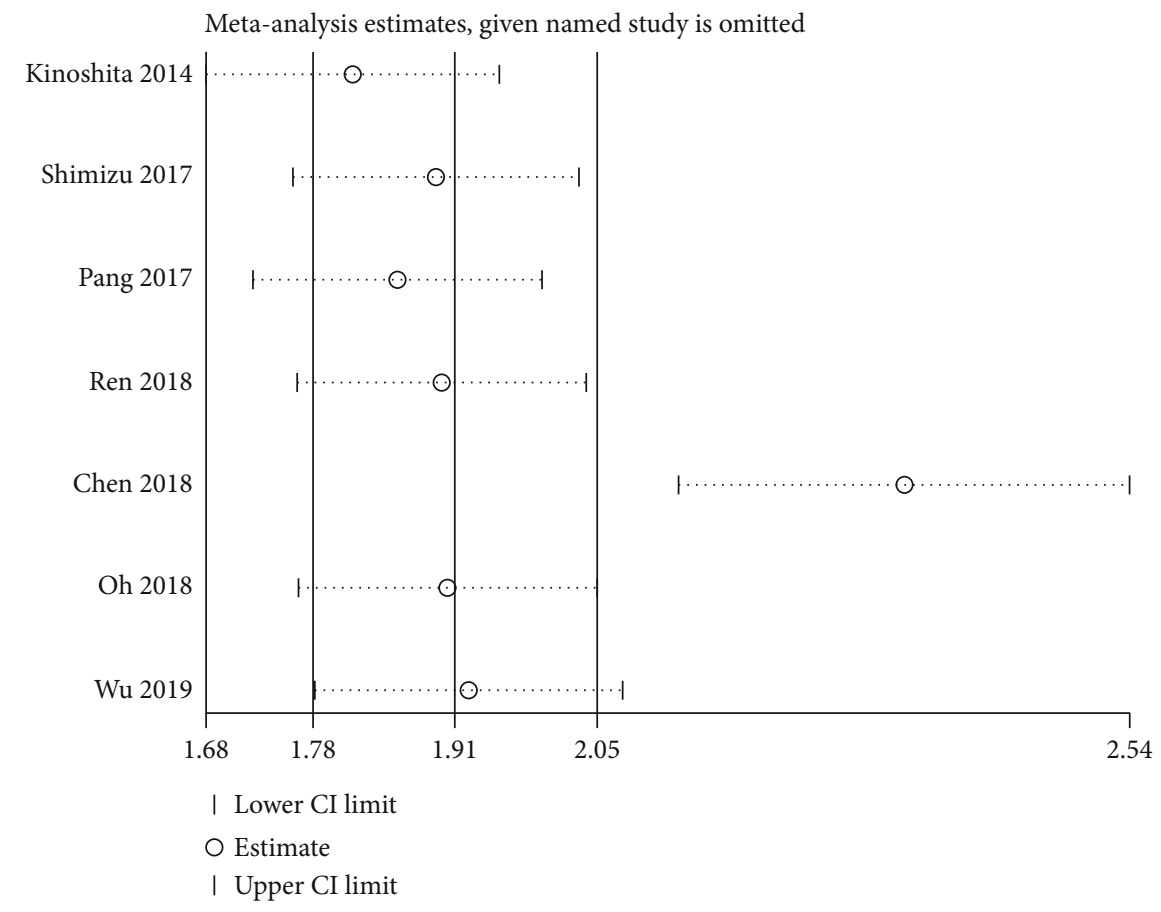

FIGURE 4: Sensitivity analysis for the pooled OS comparing between low and high CAR groups.

3.5. Sensitivity Analysis. Sensitivity analysis was conducted for the pooled OS comparing between low and high CAR groups. Results showed that the pooled OS rates comparing between low and high CAR groups did not change substantially after removal of any included study (Figure 4), which indicated that the results were considerably reliable.

3.6. Publication Bias Analysis. The publication bias analysis was conducted for the pooled HR for the OS rates comparing between low and high CAR groups, and results showed that significant publication biases were observed in the Egger's test $(P=0.02$, Figure 5$)$, but no significant publication biases were observed in OS comparing between high and low level of CAR using the Begg's test $(P=0.23)$. Trim and fill method was conducted to assess the stability of the result. After "trim and fill" analysis, four more studies were enrolled, and the pooled HR for the pooled OS rates comparing between low and high CAR groups was 1.744 (1.221-2.267), which indicated that the unpublished studies would not change the results. Funnel plot after adjusted was shown in Figure 6.

\section{Discussion}

This is the first meta-analysis addressing the prognostic value of CAR in HCC. Seven studies were eligible including 202 patients, and results showed that CAR was not only correlated with clinicopathological characteristics but was also associated with OS of patients with HCC. What's more, the results were confirmed by subgroup analysis and sensitivity analysis. Hence, CAR could be served as a prognostic biomarker for patients with HCC. 


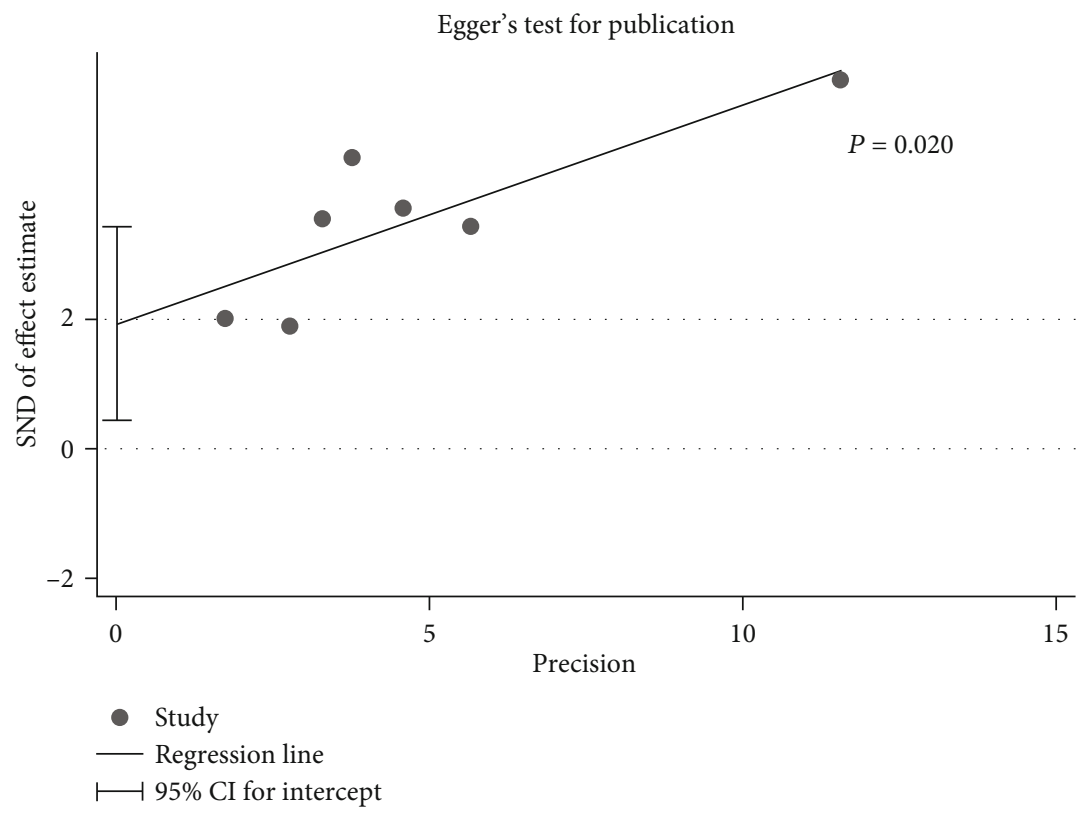

FIGURE 5: Egger's test for publication bias.

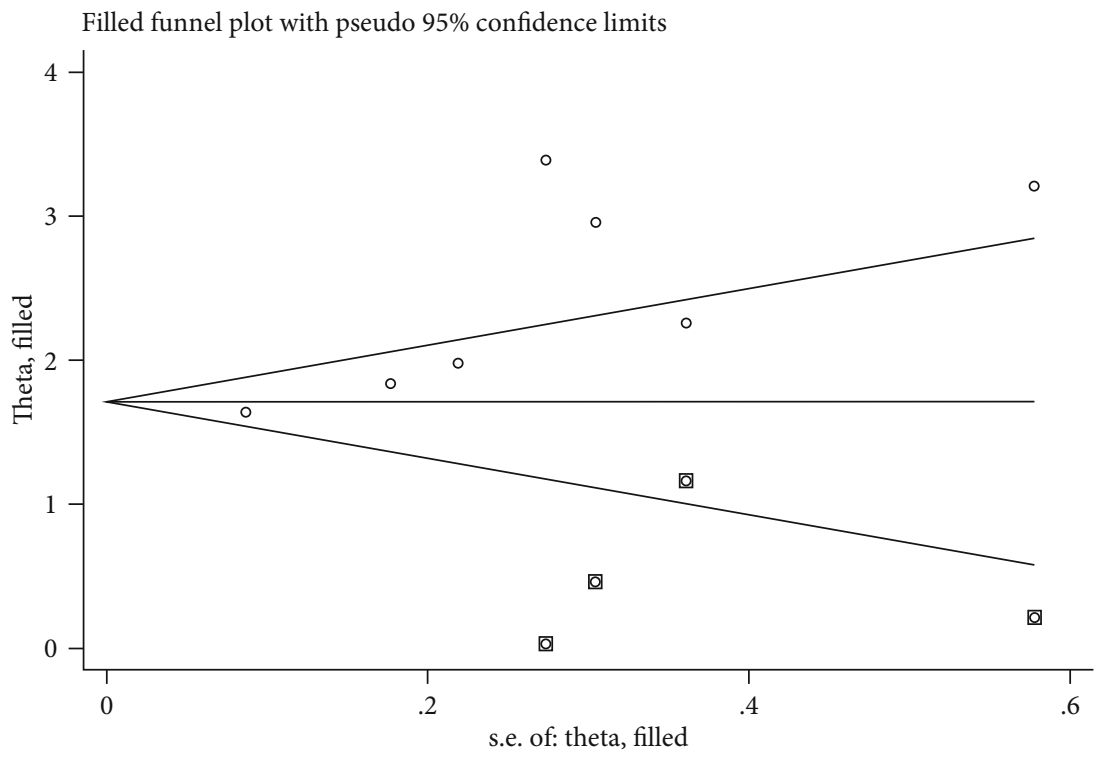

FIGURE 6: Funnel plot after adjusted by trim and fill method.

As noninvasive and obtained easily in clinic indicators, serum biological markers have always been considered as the ideal biomarkers for the prognosis in tumor [22, 23]. CAR, as a star prognostic biomarker for cancer [24, 25], two of whose elements, CRP and ALB, are derived from blood. Recently, CAR has been confirmed by several metaanalysis in colorectal cancer [12], esophageal cancer [11], and nasopharyngeal cancer [10], except hepatocellular carcinoma. In this meta-analysis, we found that high CAR was associated with poor prognosis for all patients with HCC. As for patients receiving surgery, high CAR was associated with poor OS and DFS, which indicated that CAR could be applied widely.
The roles of CAR go far beyond the prediction of prognosis. AFP is the most common indicator used to screen early HCC $[26,27]$; in this study, we found that the level of CAR was closely associated with the concentration of AFP, indicating that CAR could be served as a biomarker for HCC screening. Tumor number $[28,29]$ and tumor diameter are the two important indexes for HCC staging, and high CAR were confirmed to be associated with more tumor sites and bigger ones, suggesting that CAR could be a predictor for tumor staging.

However, mechanism underlying the prognostic value of CAR in HCC remains unclear [30, 31]. Potential explanations might be as follows: (1) HCC is a classical inflammation- 
related cancer, which is often progressed from hepatitiscirrhosis $[4,32]$, and nonsteroidal anti-inflammatory drugs such as aspirin are confirmed to be able to reduce the risk of morbidity and mortality of HCC [33, 34]; (2) the role of nutrition status is tended to be more and more important in the prognosis of cancer, especially in advanced cancers, and as a direct index of nutrition status, ALB is only synthesized in liver $[15,35,36]$. Hence, in our opinion, the prognostic value of CAR is much bigger in HCC than that in other solid tumors.

There were several limitations in this study. First, all the included studies were retrospective studies, indicating an obvious recalling bias. Second, all the included studies came from Japan, South Korea, and China, indicating an apparent regional bias because the epidemiology differed between the West and East. Third, patients receiving surgery or not were enrolled into four of the included studies, indicating obvious confounding bias. Fourth, the cut-off value of CAR varied from each included study. Fifth, dynamic changes in CAR were considered to be much more meaningful than pretreatment CAR, but only one study [18] on this topic was identified. The last but not the least, publication bias was hard to be avoided, although significant publication bias was not detected after "trim and fill" analysis.

\section{Conclusion}

With the current data, we concluded without questions that CAR was closely correlated with prognosis of patients with HCC and could be applied as a noninvasive prognostic biomarker for HCC in clinic. In future, the cut-off value of CAR should be determined. However, given the restrictions mentioned above, multicenter, larger sample, and prospective randomized trials are warranted to confirm this metaanalysis.

\section{Data Availability}

Answer: Comment: N/A

\section{Conflicts of Interest}

The authors deny any conflict of interest.

\section{Authors' Contributions}

Jingrong Li, Nanping Lin, and Qiao Ke performed the acquisition of data and analysis and interpretation of data; Lei Wang performed the acquisition of data, analysis and interpretation of data, drafted the article, and performed the final approval; Yingping Cao and Jingfeng Liu performed the conception and design of the study, critical revision, and final approval.

\section{Acknowledgments}

Thanks to Professor Xiaolong Liu, from the United Innovation of Key Laboratory of Fujian Province, for editing the English text of this manuscript. This study was supported by the Startup Fund for Scientific Research, Fujian Medical University (Grant number: 2018QH1195).

\section{References}

[1] F. Bray, J. Ferlay, I. Soerjomataram, R. L. Siegel, L. A. Torre, and A. Jemal, "Global cancer statistics 2018: GLOBOCAN estimates of incidence and mortality worldwide for 36 cancers in 185 countries," CA: a Cancer Journal for Clinicians, vol. 68, no. 6, pp. 394-424, 2018.

[2] K. Ebisawa, Y. Midorikawa, T. Higaki et al., "Natural history of nonenhancing lesions incidentally detected during the diagnosis of hepatocellular carcinoma," Surgery, vol. 160, no. 3, pp. 654-660, 2016.

[3] R. Lencioni, "Loco-regional treatment of hepatocellular carcinoma," Hepatology, vol. 52, no. 2, pp. 762-773, 2010.

[4] H. B. El-Serag and K. L. Rudolph, "Hepatocellular carcinoma: epidemiology and molecular carcinogenesis," Gastroenterology, vol. 132, no. 7, pp. 2557-2576, 2007.

[5] A. B. Benson, M. I. D'Angelica, D. E. Abbott et al., "NCCN guidelines insights: hepatobiliary cancers, version 1.2017," Journal of the National Comprehensive Cancer Network, vol. 15 , no. 5, pp. 563-573, 2017.

[6] J. Zhou, H. C. Sun, Z. Wang et al., "Guidelines for diagnosis and treatment of primary liver cancer in China (2017 Edition)," Liver Cancer., vol. 7, no. 3, pp. 235-260, 2018.

[7] X. Yu, Y. Wen, Y. Lin et al., "The value of preoperative Glasgow prognostic score and the C-reactive protein to albumin ratio as prognostic factors for long-term survival in pathological T1N0 esophageal squamous cell carcinoma," Journal of Cancer, vol. 9, no. 5, pp. 807-815, 2018.

[8] Y. Wang, L. Yang, L. Xia, and Y. Chen, "High C-reactive protein/albumin ratio predicts unfavorable distant metastasis-free survival in nasopharyngeal carcinoma: a propensity scorematched analysis," Cancer Management and Research, vol. 10, pp. 371-381, 2018.

[9] M. Shibutani, H. Nagahara, T. Fukuoka et al., "Prognostic significance of the C-reactive protein-to-albumin ratio in patients with metastatic colorectal cancer treated with Trifluridine/thymidine phosphorylase inhibitor as later-line chemotherapy," Anticancer Research, vol. 39, no. 2, pp. 1051-1057, 2019.

[10] N. Gao, R. N. Yang, Z. Meng, and W. H. Wang, "The prognostic value of C-reactive protein/albumin ratio in nasopharyngeal carcinoma: a meta-analysis," Bioscience Reports, vol. 38, no. 6,2018

[11] Y. Zhang, G. Xiao, and R. Wang, "Clinical significance of systemic immune-inflammation index (SII) and C-reactive protein-to-albumin ratio (CAR) in patients with esophageal cancer: a meta-analysis [J]," Cancer Management and Research, vol. 11, pp. 4185-4200, 2019.

[12] Y. Fan, S. Xiang, Z. Dai, C. Zou, X. Wang, and Z. Gao, "Prognostic significance of $\mathrm{C}$-reactive protein to albumin ratio in colorectal cancer patients: a meta-analysis," International Journal of Colorectal Disease, vol. 34, no. 6, pp. 1105-1111, 2019.

[13] A. Kinoshita, H. Onoda, N. Imai et al., "The C-reactive protei$\mathrm{n}$ /albumin ratio, a novel inflammation-based prognostic score, predicts outcomes in patients with hepatocellular carcinoma," Annals of Surgical Oncology, vol. 22, no. 3, pp. 803-810, 2015.

[14] S. Pang, Z. Zhou, X. Yu et al., "The predictive value of integrated inflammation scores in the survival of patients with 
resected hepatocellular carcinoma: a retrospective cohort study," International Journal of Surgery, vol. 42, pp. 170-177, 2017.

[15] T. Shimizu, M. Ishizuka, T. Suzuki et al., "The value of the Creactive protein-to-albumin ratio is useful for predicting survival of patients with child-Pugh class A undergoing liver resection for hepatocellular carcinoma," World Journal of Surgery, vol. 42, no. 7, pp. 2218-2226, 2018.

[16] T. Oh, Y.-R. Choi, J. Cho, Y.-S. Yoon, H.-S. Han, and J.-H. R. In Park, "The High-Sensitivity C-Reactive Protein/Albumin Ratio Predicts Long-Term Oncologic Outcomes after Curative Resection for Hepatocellular Carcinoma," Journal of Clinical Medicine, vol. 7, no. 6, p. 139, 2018.

[17] J. Chen, A. Fang, M. Chen et al., "A novel inflammation-based nomogram system to predict survival of patients with hepatocellular carcinoma," Cancer Medicine, vol. 7, no. 10, pp. 50275035, 2018.

[18] Y. Ren, X. Fan, G. Chen, D. Zhou, H. Lin, and X. Cai, "Preoperative C-reactive protein/albumin ratio to predict mortality and recurrence of patients after curative resection with hepatocellular carcinoma," Medicina Clínica (Barcelona), vol. 153, no. 5, pp. 183-190, 2018.

[19] M. T. Wu, S. Y. He, S. L. Chen et al., "Clinical and prognostic implications of pretreatment albumin to C-reactive protein ratio in patients with hepatocellular carcinoma," BMC Cancer, vol. 19, no. 1, p. 538, 2019.

[20] D. Moher, A. Liberati, J. Tetzlaff, and D. G. Altman, "Preferred reporting items for systematic reviews and meta-analyses: the PRISMA statement," Journal of Clinical Epidemiology, vol. 62, no. 10, pp. 1006-1012, 2009.

[21] L. Wang, N. Lin, F. Xin, Q. Ke, Y. Zeng, and J. Liu, "A systematic review of the comparison of the incidence of seeding metastasis between endoscopic biliary drainage and percutaneous transhepatic biliary drainage for resectable malignant biliary obstruction," World Journal of Surgical Oncology, vol. 17, no. 1, p. 116, 2019.

[22] A. Mantovani, P. Allavena, A. Sica, and F. Balkwill, "Cancerrelated inflammation," Nature, vol. 454, no. 7203, pp. 436444, 2008.

[23] C. I. Diakos, K. A. Charles, D. C. McMillan, and S. J. Clarke, "Cancer-related inflammation and treatment effectiveness," The Lancet Oncology, vol. 15, no. 11, pp. e493-e503, 2014.

[24] S. Ide, Y. Toiyama, Y. Okugawa et al., "Clinical significance of C-reactive protein-to-albumin ratio with rectal cancer patient undergoing chemoradiotherapy followed by surgery," Anticancer Research, vol. 37, no. 10, pp. 5797-5804, 2017.

[25] H. Saito, Y. Kono, Y. Murakami et al., "Prognostic significance of the preoperative ratio of C-reactive protein to albumin and neutrophil-lymphocyte ratio in gastric cancer patients," World Journal of Surgery, vol. 42, no. 6, pp. 1819-1825, 2018.

[26] D. Bernuau, A. Poliard, and G. Feldmann, "In situ cellular analysis of $\alpha$-fetoprotein gene expression in regenerating rat liver after partial hepatectomy," Hepatology, vol. 8, no. 5, pp. 997-1005, 1988.

[27] H. B. El-Serag, J. A. Marrero, L. Rudolph, and K. R. Reddy, "Diagnosis and treatment of hepatocellular carcinoma," Gastroenterology, vol. 134, no. 6, pp. 1752-1763, 2008.

[28] K. Katayama, T. Imai, Y. Abe et al., "Number of nodules but not size of hepatocellular carcinoma can predict refractoriness to transarterial chemoembolization and poor prognosis," Jour- nal of Clinical Medical Research, vol. 10, no. 10, pp. 765-771, 2018.

[29] K. S. Jeng, C. C. Huang, C. K. Lin et al., "Reappraisal of failures in downstaging treatment of hepatocellular carcinoma prior to liver transplant-preliminary report on the impact of underestimations of tumor numbers and tumor sizes as measured from imaging before transplant," Transplantation Proceedings, vol. 51, no. 5, pp. 1428-1434, 2019.

[30] R. W. C. Pang and R. T. P. Poon, "novel prognostic biomarkers in hepatocellular carcinoma," Nature Reviews. Gastroenterology \& Hepatology, vol. 9, no. 12, pp. 691-692, 2012.

[31] B. Hu, X. R. Yang, Y. Xu et al., "Systemic immuneinflammation index predicts prognosis of patients after curative resection for hepatocellular carcinoma," Clinical Cancer Research, vol. 20, no. 23, pp. 6212-6222, 2014.

[32] Z. Lei, J. Li, D. Wu et al., "Nomogram for preoperative estimation of microvascular invasion risk in hepatitis B virus-related hepatocellular carcinoma within the milan criteria," JAMA Surgery, vol. 151, no. 4, pp. 356-363, 2016.

[33] T. G. Simon, Y. Ma, J. F. Ludvigsson et al., "Association between aspirin use and risk of hepatocellular carcinoma," JAMA Oncology, vol. 4, no. 12, pp. 1683-1690, 2018.

[34] T. Y. Lee, Y. C. Hsu, H. C. Tseng et al., "Association of daily aspirin therapy with risk of hepatocellular carcinoma in patients with chronic hepatitis B," JAMA Internal Medicine, vol. 179, no. 5, pp. 633-640, 2019.

[35] T. Okabayashi, I. Nishimori, T. Sugimoto et al., "Effects of branched-chain amino acids-enriched nutrient support for patients undergoing liver resection for hepatocellular carcinoma," Journal of Gastroenterology and Hepatology, vol. 23, no. 12, pp. 1869-1873, 2008.

[36] K. Ichikawa, T. Okabayashi, H. Maeda et al., "Oral supplementation of branched-chain amino acids reduces early recurrence after hepatic resection in patients with hepatocellular carcinoma: a prospective study," Surgery Today, vol. 43, no. 7, pp. 720-726, 2013. 